\title{
A Large Hospital Outbreak of Klebsiella pneumoniae (DHA-1 and SHV-11 Positive): Importance of Detection and Treatment of ampC $\beta$-Lactamases
}

\author{
Timothy Vanwynsberghe ${ }^{\mathrm{a}}$, Katia Verhamme ${ }^{\mathrm{b}}$, Marijke Raymaekers ${ }^{\mathrm{c}}$, Reinoud Cartuyvels ${ }^{\mathrm{c}}$, \\ Kristien Van Vaerenbergh ${ }^{\mathrm{a}}$, An Boel ${ }^{\mathrm{a}}$ and Hans de Beenhouwer ${ }^{*}$,a
}

\author{
${ }^{a}$ Clinical Microbiology, OLV Hospital, Aalst; ${ }^{b}$ Infection control and epidemiology, OLV Hospital, Aalst; ${ }^{c}$ Clinical \\ Microbiology, Virga-Jesse Hospital, Hasselt, Belgium
}

\begin{abstract}
Plasmid-borne ampC $\beta$-lactamases are emerging worldwide. There is no gold standard in detecting them so it is presumed their prevalence is underestimated. They may confer resistance to broad-spectrum cephalosporins. In this paper the first outbreak in Europe of a Klebsiella pneumoniae harbouring a plasmid-borne DHA-1 $\beta$-lactamase and a SHV-11 $\beta$ lactamase is reported. Following CLSI (Clinical Laboratories Standards Institute) guidelines the majority of isolates would have been reported susceptible to third generation cephalosporines in spite of increased MIC values. No failures were observed in patients treated with cefepime or meropenem. Infections were linked to stay on the intensive care unit and/or urological interventions. The outbreak was stopped after a meeting was organised between the department of infection control of the hospital and the complete ICU and urology staff in which the importance of strict hand hygiene to limit transmission was stressed.
\end{abstract}

Keywords: $\beta$-lactamases, AmpC, DHA-1, SHV-11, Cefepime treatment, K. pneumonia.

\section{INTRODUCTION}

In the ever evolving world of bacterial resistance to antibiotics, one of the more frequent and powerful weapons of bacteria is the production of $\beta$-lactamases. In Gramnegatives this is the most important way of bacterial resistance against $\beta$-lactam antibiotics. These bacterial enzymes hydrolyze the $\beta$-lactam ring and inactivate the $\beta$ lactam antibiotic before it reaches the penicillin-binding protein (PBP) [1-3]. The $\beta$-lactamases are structurally very similar to PBP. Their active site contains either a serine residue or a metal ion which together with the conformation of that active site determines the substrate. Based on the substrate Ambler has made a classification of $\beta$-lactamases. In this classification with four classes, Ambler class $C$ consists of cephalosporinases that hydrolyze cephalosporins more effectively than penicillins [4]. These plasmid-borne or chromosomal enzymes are often called ampC $\beta$-lactamases due to the specific activation of the ampC gene. Genes encoding ampC $\beta$-lactamases are much more frequently chromosomal than plasmid-mediated. K. pneumoniae is one of the few Gram-negative bacteria which do not possess a chromosomal ampC $\beta$-lactamase, but can acquire these enzymes via transfer of ampC-containing plasmids. In this article an outbreak of $K$. pneumoniae with a plasmid-borne ampC $\beta$-lactamase in a Belgian hospital is described. The importance of antibiotic resistance and treatment, and techniques of detection of acquired ampC enzymes is discussed.

Preliminary accounts of this work have been presented previously as a short report [5].

*Address correspondence to this author at the OLV hospital, Moorselbaan 164, 9300 Aalst, Belgium; Tel: 32053724291; Fax: +32053724588;

E-mail: hans.de.beenhouwer@olvz-aalst.be

\section{MATERIALS AND METHODS}

Routine identification and antibiotic susceptibility testing were performed by Phoenix ${ }^{\circledR}$ (BD). Clinical isolates identified as $K$. pneumoniae and flagged as "possible extended-spectrum $\beta$-lactamases (ESBL) positive" were investigated with our modified double-disk synergy test. Flagging is based on a combination of $\mathrm{BD}$ and home made rules.

Modified Double-Disk Synergy Test (DDST): Double disk testing according to Jarlier [6] with amoxicillinclavulanic acid, ceftazidime, ceftriaxone, supplemented with aztreonam, cefepime, and cefotaxime was performed as ESBL confirmation assay. An extra cefoxitin disk was added as a screening measure for ampC $\beta$-lactamases [7].

AmpC Disk Test: presented by Black et al. [8], was performed in order to verify the presence of an ampC $\beta$ lactamase. EDTA makes the bacterial wall permeable to the ampC $\beta$-lactamase resulting in enzymatic desactivation of cefoxitin. Visually a flattening or indentation of the zone of the cefoxitin-susceptible E. coli is seen (Fig. 1).

18 clinical isolates were tested by PCR and pulse field gel electrophoresis.

DNA Macrorestriction and PFGE Analysis: DNA macrorestriction using the XBA1 restriction enzyme (Genepath group 6 reagent kits, Bio-Rad, Hercules, USA) was performed according to the manufacturer's instructions. PFGE restriction patterns were obtained on a GenePath system (Bio-Rad, Hercules, USA). The PFGE patterns were analysed and clustered into dendrograms with the Fingerprinting II software (Bio-Rad, Hercules, USA). Interpretation was done using the criteria of Tenover et al. [9]. 


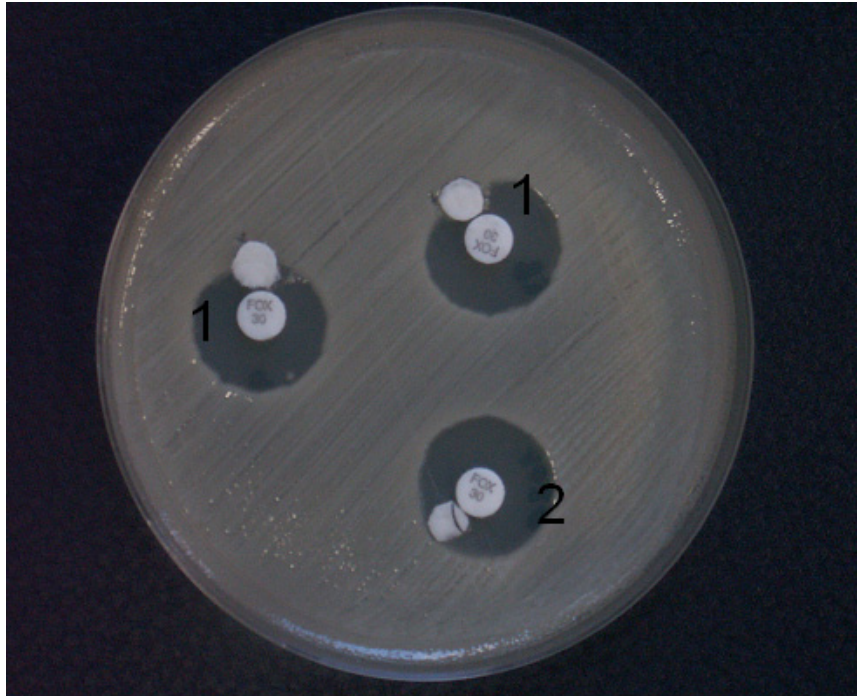

Fig. (1). AmpC disk test: indentation towards cefoxitin points to the presence of an AmpC $\beta$-lactamase. The test strain is applied on a paper disk containing TRIS-EDTA and is placed near a cefoxitin (FOX) disk. (1: isolate nr 67224, 2: negative control).

\section{Polymerase Chain Reaction (PCR)}

DNA Preparation. Bacterial solutions were boiled for 10 min. PCR experiments were performed with these crude lysates.

Amplification of $\boldsymbol{\beta}$-Lactamase (BLA) Genes: Strains suspected of ESBL production (based on DDST) were examined for the presence of $b l a_{T E M}, b l a_{S H V}$ and $b l a_{C T X-M}$ by PCR using sequence specific primers. The gene encoding the $16 \mathrm{~s}$ rRNA was used as an amplification control. PCR products were separated on a $1.5 \%$ agarose gel stained with ethidium bromide. Isolates positive for $b l a_{T E M}$ were additionally examined for the presence of bla ${ }_{T E M-24}$ with sequence specific primers (Table 1).

Sequencing of BLA Genes: PCR products positive with the consensus primers that were previously described, but negative for $b l a_{T E M-24}$, were used for direct sequencing. Dye terminator cycle sequencing was performed and analysis was performed on a CEQ8000 Genetic Analysis System (Beckman Coulter, Fullerton, CA, USA). The obtained sequences were compared with sequences in Genbank (National Centre for Biotechnology
Information) and with sequences for TEM, SHV and CTX-M extended spectrum $\beta$-lactamases ([10], Available from: URL: http://www.ncbi. nih.gov/BLAST).

The Presence of DHA-1 was confirmed by G. A. Jacoby (Lahey Clinic, Burlington, USA). A PCR product was seen for DHA-1 primers but not for ACT-1, CMY, FOX, or MIR-1 $\beta$ lactamases.

\section{Epidemiology Data on ampC Positive K. pneumoniae}

From the $1^{\text {st }}$ August 2006 on, the department of Infection Control and Hospital epidemiology was informed, by daily mail, on each sample harbouring an ampC positive $K$. pneumoniae. The lab results and medical charts for the respective patients were carefully reviewed and all data were collected in a database. This database contained information on patient demographics, medical history, medical interventions, microbiology and antimicrobial treatment. Descriptive statistical analysis was performed using SPSS 15.0. The Kolmogorov-Smirnov test was used to assess normality. The difference in distribution of sample site location for both ampC positive and ampC negative $K$. pneumoniae was tested by means of a two-tailed ${ }_{\chi}{ }^{2}$ test. The results were considered statistically significant at a p-value of $<0.05$.

Meetings with all involved partners (medical wards, department of microbiology, department of infection control and hospital epidemiology) were held at regular basis to discuss the collected data and work out preventive measures.

\section{RESULTS}

\section{Phenotype}

The 42 strains (isolated from August 2006 till July 2007) of K. pneumoniae shared the same phenotype (Fig. 2). All were cefoxitin-resistant $(\varnothing=6 \mathrm{~mm})$. Amoxicillin-clavulanic acid (AMC) induced resistance for all $\beta$-lactams except cefepime. Scattered colonies were seen in the inhibition zones of ceftazidime, ceftriaxone, aztreonam, cefotaxime, and AMC, but again not for cefepime. The cefepime inhibition zone showed a small enhancement (phantom zone) near AMC.

The MIC values for the third and fourth generation cephalosporins respectively ranged from 4 to $>16 \mu \mathrm{g} / \mathrm{ml}$ for ceftazidime, $\leq 2$ to $4 \mu \mathrm{g} / \mathrm{ml}$ for ceftriaxone, and $\leq 1$ to 16 $\mu \mathrm{g} / \mathrm{ml}$ for cefepime. MIC values for aztreonam ranged from

\section{Table 1. PCR Primers}

\begin{tabular}{|c|c|c|c|}
\hline Gene & Primer Sequence (5'-3') & Amplicon Length (bp) & Annealing Temperature $\left({ }^{\circ} \mathrm{C}\right)$ \\
\hline bla $_{\text {TEM }}$ & $\begin{array}{l}\text { ATGAGTATTMAACATTTCCG } \\
\text { CCAAWGCTTAATCAGTGAGG }\end{array}$ & 858 & 50 \\
\hline bla $_{T E M}$ & $\begin{array}{l}\text { ATGGATCCTCAACATTTCCG } \\
\text { CCAAWGCTTAATCAGTGAGG }\end{array}$ & 858 & 50 \\
\hline$b^{b l a} a_{S H V}$ & $\begin{array}{l}\text { ATGCGTTWTDTTCGCCTGTG } \\
\text { AGCGTTGCCAGTGCTCGATC }\end{array}$ & 859 & 58 \\
\hline bla $_{C T X-M}$ & $\begin{array}{l}\text { SCVATGTGCAGYACCAGTAA } \\
\text { ACCAGAAYVAGCGGBGC }\end{array}$ & 570 & 58 \\
\hline bla $_{\text {TEM-24 }}$ & $\begin{array}{l}\text { GGGCAAGAGCAACTCGGT } \\
\text { AGACCCACGCTTACCGGT }\end{array}$ & 470 & 58 \\
\hline
\end{tabular}


$\leq 2$ to $8 \mu \mathrm{g} / \mathrm{ml}$ (Table 2 ).

Based on the phenotype it was very likely that these strains expressed an inducible plasmid-mediated ampC $\beta$ lactamase, suggesting the DHA-type [11, 12]. In these strains the ampC disk test was used which was positive pointing to the presence of an ampC $\beta$-lactamase (Fig. 1).

\section{PCR and PFGE}

PCR using consensus primers (Table 1) and sequencing of the BLA gene showed the presence of the $b a_{S H V-1 I}$ gene, which is not classified as an ESBL-producing gene. Another polymerase chain reaction in order to detect ampC $\beta$ lactamases, revealed a DHA-1-like product. Besides that a qnrB gene which confers resistance to quinolones was found (GA Jacoby, personal communication).

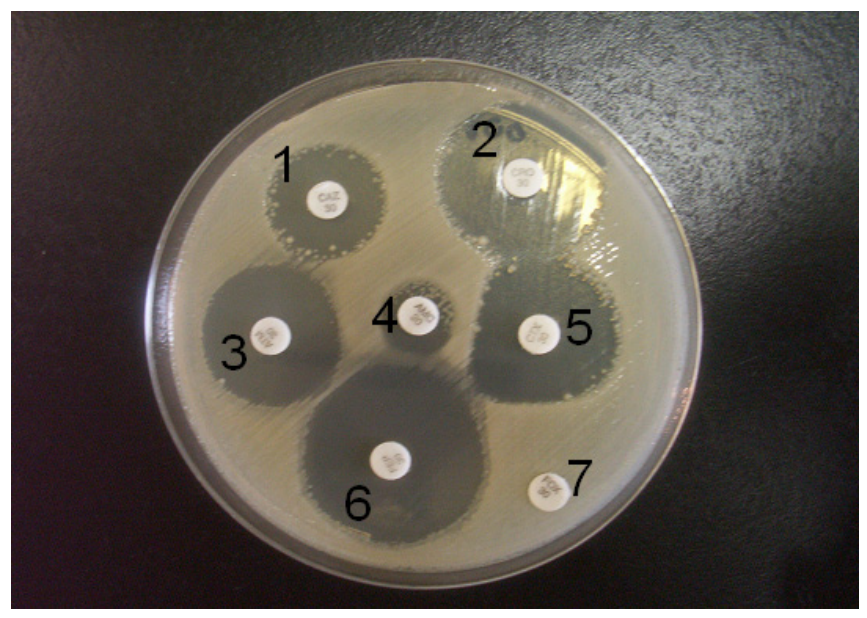

Fig. (2). Phenotype of one of the isolates on our modified doubledisk synergy test (1: ceftazidime, 2: ceftriaxone, 3: aztreonam, 4: amoxicillin-clavulanic acid, 5: cefotaxime, 6: cefepime, and 7: cefoxitin).

Pulse field gel electrophoresis gave 3 types. All strains were 'closely' or 'possibly related' to the outbreak according to the Tenover criteria [9]. This suggests a single clonal spread.

\section{Epidemiology}

A retrospective analysis was performed on all clinical isolates that were identified in our hospital from the beginning of 2004 until the $1^{\text {st }}$ August 2006. Based on identification and antimicrobial susceptibility data available in the Phoenix database, this analysis revealed that in 2004, only 8 of the $264 \mathrm{~K}$. pneumoniae isolates had a similar antibiotic profile as the ampC $\beta$-lactamase positive $K$. pneumoniae. A similar antibiotic profile was identified in $9 / 287 \mathrm{~K}$. pneumoniae isolates in 2005 and in $10 / 130$ in the first semester of 2006. Unfortunately, none of these strains was available for genotypic/phenotypic analysis.

From August 2006 until July 2007, the incidence of $K$. pneumoniae with the above described phenotype was prospectively monitored. During this period, a total of 42 patients were identified harbouring an identical strain and patient characteristics are given in Supplemental Data 1. The mean age of the patients was 72.7 years (std 9.7) and the male to female ratio was 1.8 . More than $30 \%$ of the patients were staying at the ICU at the time of isolation, followed by a ward of internal medicine $(21 \%)$ and a surgical ward $(19 \%)$. AmpC positive K. pneumoniae was isolated in 6 outpatients $(14 \%)$. The median number of days of hospitalisation until isolation of ampC $\beta$-lactamase positive K. pneumoniae was 9.5 days (IQR 22 days). The majority of first isolates came from urine samples $(27 / 42-64 \%)$ followed by pus samples $(7 / 42-17 \%)$ and sputum samples $(5 / 42-12 \%)$. This distribution of sample sites corresponds to the distribution within our hospital of ampC negative $K$. pneumoniae.

14 patients had subsequent positive samples for ampC positive K. pneumoniae - for the majority of these patients, $K$. pneumoniae was isolated from the same source as the first positive isolate. Two patients were diagnosed with a urinary colonization of ampC positive $K$. pneumoniae. AmpC positive $K$. pneumoniae isolation was related to ICU stay: $30 \%(13 / 42)$ of patients were staying at the ICU at the time of isolation and $57 \%(24 / 42)$ of patients had stayed at the ICU department in the 30 days prior to the isolation of ampC positive K. pneumoniae.

Environmental samples were taken at the ICU. In none of these samples ampC positive K. pneumoniae was detected.

\section{Treatment}

Of the patients treated for a clinically relevant (fever, elevated C-reactive protein, leukocytosis) ampC positive $K$. pneumoniae $(\mathrm{n}=21), 43 \%$ received meropenem $(\mathrm{n}=9)$ and $29 \%$ were treated with cefepime $(\mathrm{n}=6)$. Treated infections were the following: urinary tract infections $(\mathrm{n}=13)$, pneumonia $(\mathrm{n}=2)$, sepsis $(\mathrm{n}=2)$, wound infections $(\mathrm{n}=2)$, peritonitis $(\mathrm{n}=2)$. Of the admitted patients $(\mathrm{n}=36), 8$ patients died (22\%), of which 2 deaths could be attributed to the infection with $K$. pneumoniae. The latter two patients received respectively either no therapy or ciprofloxacine (to which the ampC K. pneumoniae was resistant). All patients who did receive meropenem or cefepime had a good outcome with in most patients a proven eradication (Table 3).

\section{DISCUSSION}

Many gram-negative bacteria possess an ampC gene on their chromosome. The only known exceptions are

Table 2. Spread and Median of the MIC's of 45 Isolates of $K$. pneumoniae (ampC) for Amoxicillin-Clavulanic Acid (AMC), Aztreonam (ATM), Ceftazidime (CAZ), Ceftriaxone (CRO), Cefepime (FEP), Ciprofloxacine (CIP), and PiperacillinTazobactam (TZP)

\begin{tabular}{|c|c|c|c|c|c|c|c|}
\hline $\mathbf{n}=\mathbf{4 5}$ & AMC & ATM & CAZ & CRO & FEP & TZP & CIP \\
\hline \hline MIC spread $(\mu \mathrm{g} / \mathrm{ml})$ & $16->16$ & $\leq 1->16$ & $4->16$ & $\leq 2-4$ & $\leq 1-16$ & $8->64$ & $>2$ \\
\hline MIC median $(\mu \mathrm{g} / \mathrm{ml})$ & $>16$ & 2 & 8 & $\leq 2$ & 2 & 64 & $>2$ \\
\hline
\end{tabular}


Table 3. Outcome of Antibiotic Therapy of 20 Treated Patients

\begin{tabular}{|c|c|c|c|}
\hline $\mathbf{n}=\mathbf{2 0}$ & Good Outcome (Eradication) & $\begin{array}{c}\text { Unclear Outcome (Lost to Follow-Up, Early Antibiotic } \\
\text { Switch, no Eradication) }\end{array}$ & Negative Outcome (Death) \\
\hline \hline Meropenem & $5(71 \%)$ & $2(29 \%)$ & 0 \\
\hline Cefepime & $3(60 \%)$ & $2(40 \%)$ & 0 \\
\hline Temocillin & $1(50 \%)$ & $1(50 \%)$ & $1(50 \%)$ \\
\hline Ciprofloxacin & 0 & $2(100 \%)$ & 0 \\
\hline Amikacin & 0 & $1(100 \%)$ & 0 \\
\hline Piperacillin-tazobactam & 0 & $1(100 \%)$ & 0 \\
\hline Phosphomycin & 0 & & 0 \\
\hline
\end{tabular}

Klebsiella spp, Salmonella spp, and Proteus mirabilis [13, 14]. In clinically important gram-negatives that produce an ampC this $\beta$-lactamase is usually repressed. AmpC expression results from a system including ampD (a cytosolic amidase), ampG (a channel), ampR (an activator/repressor), and intermediates in peptidoglycan recycling [15]. Actions that influence these factors such as $\beta$ lactam antibiotics or a mutation, can induce ampC expression.

The DHA-1 ampC $\beta$-lactamase belongs to the Morganella morganii group. It was first discovered in 1992 in Salmonella enteritidis and is named by its site of discovery: the Dharan hospital in Saudi Arabia [16]. The first detection of DHA-1 in K. pneumoniae was in 1998 in France [17].

Until now two other DHA enzymes are discovered, DHA-2 and DHA-3, in France and Taiwan respectively and remarkably both in $K$. pneumoniae $[18,19]$.

Strains of $K$. pneumoniae containing SHV-11 together with an AmpC $\beta$-lactamase are very rare and have been described mainly in Taiwan $[20,21]$. The literature reveals that the prevalence of plasmid-mediated ampC $\beta$-lactamases in $K$. pneumoniae is rising worldwide and strains producing these enzymes have been reported from every continent, but particularly from East-Asia [22-26]. Since they aren't searched for routinely it is assumed their prevalence is underestimated.

Nowadays there is no gold standard to detect the presence of plasmid-mediated ampC $\beta$-lactamases. The laboratories searching for these ampCs use a variety of tests. Often a cephamycin such as cefoxitin is used as a screening method since cephamycin-resistance is typical to ampC $\beta$ lactamases. It is highly sensitive but not specific. As mentioned above cefoxitin-resistance points to a few possible resistance mechanisms: porin loss; ampC $\beta$ lactamase production or carbapenemase production (metallo$\beta$-lactamase). Some exceptions are known. ACC-1 for instance, an ampC $\beta$-lactamase originating from Hafnia alvei, doesn't confer resistance to cefoxitin [27]. As strains with ampC $\beta$-lactamases are resistant to $\beta$-lactam $/ \beta$ lactamase inhibitor combinations this can differentiate them from ESBLs as long as there is no concomitant production of
ampC $\beta$-lactamase and an ESBL. This can be confirmed by using a cloxacillin-based plate in which ampC's are inhibited.

The presence of a plasmid-mediated ampC $\beta$-lactamase can be confirmed with methods as the three-dimensional test, the ampC disk test, inhibitor-based tests mostly using boronic acid, or a multiplex PCR [8, 28-30]. Of these confirmatory tests multiplex PCR is the only test that can differentiate plasmid-borne ampC $\beta$-lactamases from chromosomally ampCs. In a nice paper Doi and Paterson review the detection of ampC $\beta$-lactamases, suggesting an interesting flow chart [31].

The migration of chromosomal ampC genes into plasmids may pose a serious threat to the health care. Infections with $K$. pneumoniae strains harbouring an acquired AmpC $\beta$-lactamase are associated with a higher mortality and a high risk of therapeutic failure of thirdgeneration cephalosporins, although these strains may appear susceptible in vitro [32]. It has to be stressed that strains bearing acquired ampC $\beta$-lactamases meet CLSI criteria for ESBL screening, but present with a negative ESBL confirmation test. Since these strains aren't resistant to broad-spectrum cephalosporins using CLSI breakpoints, they are usually reported as being susceptible which might hamper treatment outcome [31].

There's some controversy about the use of fourth generation cephalosporins to treat infections with ampC $\beta$ lactamases. Most authors state these antibiotics can be used unless there is concomitant production of ESBLs [26, 33, 34]. This matches an evaluation we did at our laboratory. Scattered colonies within the ceftazidime inhibition zone proved to be highly resistant with MIC values for ceftazidime above $32 \mu \mathrm{g} / \mathrm{ml}$, but remained susceptible for cefepime (data not shown). In the limited series of patients treated with cefepime in the mentioned outbreak no therapeutic failure was noticed. On the other hand in some strains MIC values for cefepime were already elevated and an in vitro inoculum effect and in vivo ampC mutants resistant to cefepime have already been observed by others $[8,35,36]$. Therefore it may be advisable to choose therapy on the basis of MIC values (Table 2).

Finding an ampC may also be clinically important because a carbapenem resistance can develop by further mutations, in particular when there is porin loss [37, 38]. 
There seems to be a remarkable association between DHA-1 enzymes and the plasmid-mediated resistance to quinolones by qnrB genes. It has been mentioned in these isolates and it has been seen before [39].

One third of all patients in our outbreak had a medical history of an urological intervention (urethral catheter excluded) in the 6 months prior to the isolation of ampC positive $K$. pneumoniae. Because of the association found between ampC positive $K$. pneumoniae isolates and a stay at the ICU and/or urological interventions, a meeting was organised between the department of infection control of the hospital and the complete ICU and urology staff. The outbreak of ampC positive $K$. pneumoniae was discussed and the importance of strict hand hygiene to limit transmission was stressed. After this meeting no more positive cases were observed.

\section{CONCLUSION}

The first outbreak in Europe with a $K$. pneumoniae harbouring a plasmid-borne DHA-1 ampC $\beta$-lactamase and SHV-11 is described. Such plasmid-borne ampCs are emerging in gram-negatives worldwide.

In the above mentioned outbreak the infections with $K$. pneumoniae were related to stay in the intensive care unit and/or history of urological conditions. The data were too few to draw meaningful conclusions with respect to antibiotic therapy. Selecting antibiotic therapy should be done carefully when dealing with $K$. pneumoniae harbouring acquired ampC $\beta$-lactamases. It may be advisable to guide therapy on the basis of MIC values when using cephalosporins. Susceptibility to carbapenems is universal. Large randomized studies are needed to clarify which antibiotics are of choice. Preventive measures are of great help in stopping these nosocomial infections.

\section{ACKNOWLEDGEMENT}

We are grateful to Professor George A. Jacoby (Lahey Clinic, Burlington, USA) for confirming the AmpC betalactamase by PCR.

\section{SUPPLEMENTARY MATERIAL}

Supplementary material is available on the publishers Web site along with the published article.

\section{REFERENCES}

[1] Babic M, Hujer AM, Bonomo RA. What's new in antibiotic resistance? Focus on $\beta$-lactamases. Drug Resist Updat 2006; 9: 142-56.

[2] Hefland MS, Bonomo RA. $\beta$-lactamases: a survey of protein diversity. Curr Drug Targets Infect Disord 2003; 3: 9-23.

[3] Rice LB, Bonomo RA. Mechanisms of resistance to antibacterial agents. In: Murray PR, Baron EJ, Jorgensen JH, Landry ML, Pfaller MA, Eds. Manual of clinical microbiology. $9^{\text {th }}$ ed. Washington DC: ASM press 2007, pp. 1114-45.

[4] Ambler RP. The structure of $\beta$-lactamases. Philos Trans R Soc Lond B Biol Sci 1980; 289: 321-31

[5] Vanwynsberghe T, Verhamme K, Raymaekers M, Cartuyvels R, Boel A, De Beenhouwer H. Outbreak of Klebsiella pneumoniae strain harbouring an AmpC (DHA-1) and a blaSHV-11 in a Belgian hospital, August-December 2006. Euro Surveill 2007; 12: e070201.3.

[6] Jarlier V, Nicolas M, Fournier G, Philippon A. Extended spectrum $\beta$-lactamases conferring transferable resistance to newer $\beta$-lactam agents in Enterobacteriaceae: hospital prevalence and susceptibility patterns. Rev Infect Dis 1988; 10: 867-78.
[7] Jacoby GA, Walsh KE, Walker VJ. Identification of extendedspectrum, AmpC, and carbapenem-hydrolyzing $\beta$-lactamases in Escherichia coli and Klebsiella pneumoniae by disk tests. J Clin Microbiol 2006; 44: 1971-6.

[8] Black JA, Moland ES, Thomson KS. AmpC disk test for detection of plasmid-mediated AmpC $\beta$-lactamases in Enterobacteriaceae lacking chromosomal AmpC $\beta$-lactamases. J Clin Microbiol 2005; 43: $3110-3$

[9] Tenover FC, Arbeit RD, Mickelsen PA, Murray BE, Persing DH, Swaminathan B. Interpreting chromosomal DNA restriction patterns produced by pulsed-field gel electrophoresis: criteria for bacterial strain typing. J Clin Microbiol 1995; 33: 2233-9.

[10] Jacoby GA, Bush K. Amino acid sequences for TEM, SHV and OXA extended spectrum and inhibitor resistant $\beta$-lactamases. 2005 [cited 2007 Dec] Available from: http://www.lahey.org/studies/webt.htm

[11] Lee K, Hong SG, Park YJ, et al. Evaluation of phenotypic screening methods for detecting plasmid-mediated AmpC $\beta$ lactamases-producing isolates of Escherichia coli and Klebsiella pneumoniae. Diagn Microbiol Infect Dis 2005; 53: 319-23.

[12] Mirelis B, Rivera A, Miró E, Mesa RJ, Navarro F, Coll P. A simple phenotypic method for differentiation between acquired and chromosomal AmpC $\beta$-lactamases in Escherichia coli. Enferm Infect Microbiol Clin 2006; 24: 370-2.

[13] Philippon A, Arlet G, Jacoby GA. Plasmid-determined AmpC-type $\beta$-lactamases. Antimicrob Agents Chemother 2002; 46: 1-11.

[14] Walther-Rasmussen J, Høiby N. Plasmid-borne AmpC $\beta$ lactamases. Can J Microbiol 2002; 48: 479-93.

[15] Jacobs C, Frère JM, Normark S. Cytosolic intermediates for cell wall biosynthesis and degradation control inducible $\beta$-lactam resistance in gram-negative bacteria. Cell 1997; 88: 823-32.

[16] Gaillot O, Clement C, Simonet M, Philippon A. Novel transferable $\beta$-lactam resistance with cephalosporinase characteristics in Salmonella enteritidis. J Antimicrob Chemother 1997; 39: 85-7.

[17] Verdet C, Boutros N, Salauze B, et al. $20^{\text {th }}$ Réunion Interdisciplinaire Chimiothérapie Anti-Infectieuse, abstr. 24/C4, 2000.

[18] Fortineau N, Poirel L, Nordmann P. Plasmid-mediated and inducible cephalosporinase DHA-2 from Klebsiella pneumoniae. J Antimicrob Chemother 2001; 47: 207-10.

[19] Wu LT, Hung SW, Chuang YC, Chen HE, Jones RN, Yu WL. Identification of a novel cephalosporinase (DHA-3) in Klebsiella pneumoniae isolated in Taiwan. Clin Microbiol Infect 2005; 11 ; 893-7.

[20] Moland ES, Hanson ND, Black JA, Hossain A, Song W, Thomson KS. Prevalence of newer $\beta$-Lactamases in gram-negative clinical isolates collected in the United States from 2001 to 2002. J Clin Microbiol 2006; 44: 3318-24.

[21] Yan JJ, Ko WC, Yung JC, Chuang CL, Wu JJ. Emergence of Klebsiella pneumoniae isolates producing inducible DHA-1 $\beta$ lactamase in a university hospital in Taiwan. J Clin Microbiol 2002; 40: 3121-6.

[22] Lee K, Lee M, Shin JH, et al. Prevalence of plasmid-mediated AmpC $\beta$-lactamases in Escherichia coli and Klebsiella pneumoniae in Korea. Microb Drug Resist 2006; 12: 44-9.

[23] Muratani T, Kobayashi T, Matsumoto T. Emergence and prevalence of $\beta$-lactamase-producing Klebsiella pneumoniae resistant to cephems in Japan. Int J Antimicrob Agents 2006; 27: 491-9.

[24] Song W, Kim JS, Kim HS, et al. Increasing trend in the prevalence of plasmid-mediated AmpC $\beta$-lactamases in Enterobacteriaceae lacking chromosomal AmpC gene at a Korean university hospital from 2002 to 2004. Diagn Microbiol Infect Dis 2006; 55: 219-24.

[25] Yan JJ, Hsueh PR, Lu JJ, et al. Extended-spectrum $\beta$-lactamases and plasmid-mediated AmpC enzymes among clinical isolates of Escherichia coli and Klebsiella pneumoniae from seven medical centers in Taiwan. Antimicrob Agents Chemother 2006; 50: 861-4.

[26] Zaidi MB, Leon V, Canche C, et al. Rapid and widespread dissemination of multidrug-resistant blaCMY-2 Salmonella Typhimurium in Mexico. J Antimicrob Chemother 2007; 60: 398401.

[27] Nadjar D, Rouveau M, Verdet C, et al. Outbreak of Klebsiella pneumoniae producing transferable AmpC-type $\beta$-lactamase (ACC1) originating from Hafnia alvei. FEMS Microbiol Lett 2000; 187: 35-40.

[28] Pai H, Seo MR, Choi TY. Association of QnrB determinants and production of extended-spectrum $\beta$-lactamases or plasmid- 
mediated AmpC $\beta$-lactamases in clinical isolates of Klebsiella pneumoniae. Antimicrob Agents Chemother 2007; 51: 366-8.

[29] Song W, Jeong SH, Kim JS, et al. Use of boronic acid disk methods to detect the combined expression of plasmid-mediated AmpC $\beta$-lactamases and extended-spectrum $\beta$-lactamases in clinical isolates of Klebsiella spp., Salmonella spp., and Proteus mirabilis. Diagn Microbiol Infect Dis 2006; 57: 315-8.

[30] Thomson KS, Sanders CC. Detection of extended-spectrum $\beta$ lactamases in members of the family Enterobacteriaceae: comparison of the double disk and three-dimensional tests. Antimicrob Agents Chemother 1992; 36: 1877-82.

[31] Doi Y, Paterson DL. Detection of plasmid-mediated class C $\beta$ lactamases. Int J Infect Dis 2007; 11: 191-7.

[32] Perez-Perez FJ, Hanson ND. Detection of plasmid-mediated AmpC $\beta$-lactamase genes in clinical isolates by using multiplex PCR. J Clin Microbiol 2002; 40: 2153-62.

[33] Pai H, Kang CI, Byeon JH, et al. Epidemiology and clinical features of bloodstream infections caused by AmpC-type- $\beta$ lactamase-producing Klebsiella pneumoniae. Antimicrob Agents Chemother 2004; 48: 3720-8.

[34] Kang CI, Pai H, Kim SH, et al. Cefepime and the inoculum effect in tests with Klebsiella pneumoniae producing plasmid-mediated
AmpC-type $\beta$-lactamase. J Antimicrob Chemother 2004; 54: 11303.

[35] Owens RC, Jr, Rice L. Hospital-based strategies for combating resistance. Clin Infect Dis 2006; 42: S173-81.

[36] Barnaud G, Benzerara Y, Gravisse J, et al. Selection during cefepime treatment of a new cephalosporinase variant with extended-spectrum resistance to cefepime in an Enterobacter aerogenes clinical isolate. Antimicrob Agents Chemother 2004; 48: $1040-2$.

[37] Mammeri H, Poirel L, Bemer P, Drugeon H, Nordmann P. Resistance to cefepime and cefpirome due to a 4-amino-acid deletion in the chromosome-encoded AmpC $\beta$-lactamase of a Serratia marcescens clinical isolate. Antimicrob Agents Chemother 2004; 48: 716-20.

[38] Alvarez M, Tran JH, Chow N, Jacoby GA. Epidemiology of conjugative plasmid-mediated AmpC $\beta$-lactamases in the United States. Antimicrob Agents Chemother 2004; 48: 533-7.

[39] Lee K, Yong D, Choi YS, et al. Reduced imipenem susceptibility in Klebsiella pneumoniae clinical isolates with plasmid-mediated CMY-2 and DHA-1 $\beta$-lactamases co-mediated by porin loss. Int J Antimicrob Agents 2007; 29: 201-6.

(C) Vanwynsberghe et al.; Licensee Bentham Open.

This is an open access article licensed under the terms of the Creative Commons Attribution Non-Commercial License (http://creativecommons.org/licenses/by$\mathrm{nc} / 3.0 /$ ) which permits unrestricted, non-commercial use, distribution and reproduction in any medium, provided the work is properly cited. 